\title{
An Integrated Selection Formulation for the Best Normal Mean: The Unequal and Unknown Variance Case
}

\author{
PINYUEN CHEN† \\ Department of Mathematics, Syracuse University, Syracuse, NY 13244-1130
}

JUN-LUE ZHANG

Department of Mathematics, Indiana Univ. of Pennsylvania, Indian, PA 15705-1072

\begin{abstract}
This paper considers an integrated formulation in selecting the best normal mean in the case of unequal and unknown variances. The formulation separates the parameter space into two disjoint parts, the preference zone $(P Z)$ and the indifference zone $(I Z)$. In the $P Z$ we insist on selecting the best for a correct selection $\left(C S_{1}\right)$ but in the $I Z$ we define any selected subset to be correct $\left(C S_{2}\right)$ if it contains the best population. We find the least favorable configuration $(L F C)$ and the worst configuration $(W C)$ respectively in $P Z$ and $I Z$. We derive formulas for $P\left(C S_{1} \mid L F C\right), P\left(C S_{2} \mid W C\right)$ and the bounds for the expected sample size $E(N)$. We also give tables for the procedure parameters to implement the proposed procedure. An example is given to illustrate how to apply the procedure and how to use the table.
\end{abstract}

Keywords: Integrated formulation; two-stage selection procedure

\section{Introduction}

This paper studies the integrated approach in selecting the best normal mean among $k$ normal populations with unequal and unknown variances. Unlike the case of common and unknown variance studied in Chen and Zhang (1997), we can not use the pooled sample variance to estimate the unknown variances in this case. One important change, compared to the case of common and unknown variance, is that in the case of unequal and unknown variances we use weighted averages as the estimators for the population means. Such change enables us to effectively evaluate the lower bounds of the probability of a correct selection.

Historically, many have studied multiple decision procedures in the case of unequal and unknown variances using the classical approaches. In the indifference zone approach, Bechhofer, Dunnett, and Sobel (1954) had men-

$\dagger$ Requests for reprints should be sent to Pinyuen Chen Department of Mathematics, Syracuse University, Syracuse, NY 13244. 
tioned the possibility of a two-stage procedure in selecting the best population among $k$ normal populations with unknown means and unequal and unknown variances. Dudewicz (1971) showed that under the indifference zone approach of Bechhofer (1954), a single-stage procedure is not appropriate in the case of unequal and unknown variances. Dudewicz and Dalal (1975) proposed a generalized Stein-type two-stage procedure using the indifference zone approach. In subset selection approach, Gupta and Huang (1974) have proposed a single-stage procedure based on unequal sample sizes for selecting a subset which would contain the best population when the variances are unknown and possibly unequal.

Chen and Sobel (1987) was the first article that proposed the integrated selection formulation. They studied a single-stage procedure for the common known variance case. The integrated formulation approach to the selection problem in the case of unequal and unknown variances has not been studied. However, such a case is important in applications since variances are often unknown and unequal in most of the real world problems. The objective of this paper is to develop a two-stage procedure, using the integrated approach, to select the best normal mean from $k$ normal populations with unequal and unknown variances.

In section 2 we state our goal, assumptions and the probability requirements. We propose a two-stage procedure in section 3 . In section 4 we derive lower bounds for the probability of a correct selection. These bounds will enable us to effectively compute the unknown parameters in our selection procedure and to guarantee the procedure to satisfy a given probability requirement $\left(P_{1}^{*}, P_{2}^{*}\right)$. The experimenter can allocate sample sizes according to these parameters. In section 5, we develop bounds for the expected sample size for the proposed procedure. The integrated formulation requires our procedure to satisfy two probability requirements simultaneously. Therefore, it is reasonable that the expected sample size in our procedure is larger than the expected sample size in the indifference zone approach. Section 6 discusses the computation of the tables. Section 7 gives an illustrative example.

\section{Assumptions, Goal, and The Probability Requirements}

Suppose that we have $k$ normal populations $\pi_{1}, \ldots, \pi_{k}$ with unknown means and unequal and unknown variances $\sigma_{1}^{2}, \sigma_{2}^{2}, \ldots, \sigma_{k}^{2}$. We denote the ordered means as $\mu_{[1]} \leq \mu_{[2]} \leq \cdots \leq \mu_{[k]}$ and denote $\pi_{(i)}$ as the population which corresponds to $\mu_{[i]}$. We also define the best population to be $\pi_{(k)}$, the population corresponding to the largest population mean $\mu_{[k]}$. 
Our goal is to derive a two-stage selection procedure $P_{E}$ which would

$$
\text { select } \pi_{(k)} \text { if } \mu_{[k]} \geq \mu_{[k-1]}+\delta^{*},
$$

or,

select a subset containing $\pi_{(k)}$ if $\mu_{[k]}<\mu_{[k-1]}+\delta^{*}$,

where $\delta^{*}>0$ is a specified constant.

We first define the parameter space as follows:

$$
\Omega=\left\{\left(\mu, \sigma^{2}\right) \mid-\infty<\mu_{i}<\infty, 0<\sigma_{i}<\infty ; i=1, \ldots, k\right\},
$$

where $\mu=\left(\mu_{1}, \ldots, \mu_{k}\right)$ and $\sigma^{2}=\left(\sigma_{1}^{2}, \ldots, \sigma_{k}^{2}\right)$.

We divide the parameter space into preference zone $(P Z)$ and indifference zone $(I Z), P Z$ and $I Z$ are defined as follows, respectively.

$$
\begin{aligned}
P Z & =\left\{(\mu, \sigma) \in \Omega \mid \mu_{[k]}-\mu_{[k-1]} \geq \delta^{*}\right\}, \\
I Z & =\left\{(\mu, \sigma) \in \Omega \mid \mu_{[k]}-\mu_{[k-1]}<\delta^{*}\right\},
\end{aligned}
$$

where $0<\delta^{*}$ is a prespecified constant.

We define $C S_{1}$ to be the event that our procedure selects the one best population when $\mu \in P Z$ and $C S_{2}$ to be the event that our procedure selects a subset that contains the best population when $\mu \in I Z$. We require that our two-stage selection procedure, $P_{E}$, which will be defined formally in Section 3, for a given $\left(P_{1}^{*}, P_{2}^{*}\right)$, would satisfy the following probability requirements:

$$
\begin{aligned}
& P\left(C S_{1} \mid P_{E}\right) \geq P_{1}^{*}, \text { and } \\
& P\left(C S_{2} \mid P_{E}\right) \geq P_{2}^{*} .
\end{aligned}
$$

\section{Procedure $P_{E}$}

We propose a Dudewicz-Dalal-type two-stage selection procedure.

Procedure $P_{E}$ :

(i) Take an initial sample $X_{i 1}, X_{i 2}, \ldots, X_{i n_{0}}$ of size $n_{0}(\geq 2)$ from population $\pi_{i} I=1,2, \ldots, k$.

Compute:

$$
\begin{aligned}
& \bar{X}_{i}\left(n_{0}\right)=\sum_{j=1}^{n_{0}} \frac{X_{i j}}{n_{0}} \\
& S_{i}^{2}\left(n_{0}\right)=\frac{1}{n_{0}-1} \sum_{j=1}^{n_{0}}\left(X_{i j}-\bar{X}_{i}\left(n_{0}\right)\right)^{2} .
\end{aligned}
$$


(ii) Define

$$
n_{i}=\max \left\{n_{0}+1,\left[\left(\frac{h^{*} S_{i}}{\delta^{*}-c}\right)^{2}\right]\right\} .
$$

[y] denotes the smallest integer greater than or equal to $y$. Here $h^{*}=$ $\max \left\{h_{1}^{*}, h_{2}^{*}\right\}$ and $h_{1}^{*}, h_{2}^{*}, h_{3}^{*}$, and $c$ are chosen to satisfy the probability requirement (5). They are the solutions of the following integral equations: When $k=2$, for given $n_{0}$ and specification $\left(\delta^{*}, P_{1}^{*}, P_{2}^{*}, a\right)$, the $h_{1}^{*}$, and $h_{2}^{*}$ values simultaneously satisfy:

$$
\begin{aligned}
& \int_{-\infty}^{+\infty} G\left(t+h_{1}^{*}\right) g(t) d t=P_{1}^{*}, \text { and } \\
& \int_{-\infty}^{+\infty} G\left(t+\frac{h_{2}^{*}}{a-1}\right) g(t) d t=P_{2}^{*}
\end{aligned}
$$

Here $G$ and $g$ are Student's $t$-distribution and density function, respectively. For any $k \geq 3$ and any $n_{0}$ and specification $\left(\delta^{*}, P_{1}^{*}, P_{2}^{*}, a\right)$, the $h_{1}^{*}, h_{2}^{*}$ and $h_{3}^{*}$ values simultaneously satisfy:

$$
\int_{-\infty}^{+\infty} G^{k-1}\left(t+h_{1}^{*}\right) g(t) d t=P_{1}^{*},
$$

and

$$
\begin{array}{rrr}
\frac{1}{k}+ & (k-1) \int_{-\infty}^{+\infty} G^{k-2}(t)\left[G\left(t+\frac{h_{2}^{*}}{(a-1)}\right)-G(t)\right] g(t) d t \\
+ & (k-1)(k-2) \int_{-\infty}^{+\infty} G^{k-3}(t)\left[G\left(t+\frac{h_{2}^{*}}{(a-1)}\right)-G(t)\right] \\
\times & & {\left[G(t)-G\left(t-h_{3}^{*}\right)\right] g(t) d t=P_{2}^{*}}
\end{array}
$$

Here $G$ and $g$ are Student's $t$-distribution and density function, respectively.

(iii) Take $n_{i}-n_{0}$ additional observations from the $i^{\text {th }}$ population. Denote the observations by $X_{i j}$, where $i=1,2, \ldots, k$ and $j=1,2, \ldots, n_{i}$. Compute:

$$
\widetilde{X}_{i}=\sum_{j=1}^{n_{i}} a_{i j} X_{i j} \quad i=1,2, \ldots, k,
$$

where $a_{i j}$ 's are to be chosen so that the following conditions are satisfied:

$$
\sum_{j=1}^{n_{i}} a_{i j}=1, a_{i 1}=a_{i 2}=, \ldots,=a_{i n_{0}},
$$


and

$$
S_{i}^{2} \sum_{j=1}^{n_{i}} a_{i j}^{2}=\left(\frac{\delta^{*}-c}{h^{*}}\right)^{2},
$$

where $i=1,2, \ldots, k$, and we use $\widetilde{X}_{[1]} \leq \widetilde{X}_{[2]} \leq, \ldots, \leq \widetilde{X}_{[k]}$ to denote the ranked $\tilde{X}$ 's.

(iv) If $\widetilde{X}_{[k]} \geq \widetilde{X}_{[k-1]}+c$, we select the population associated with $\widetilde{X}_{[k]}$. If $\widetilde{X}_{[k]}<\widetilde{X}_{[k-1]}+c$, we select a random sized subset which contains all populations $\pi_{i}$ with $\widetilde{X}_{i} \geq \widetilde{X}_{[k-1]}-d$.

Here $\delta^{*}>c, \delta^{*}=a c$, and $a>1$ is given; $h^{*}=\max \left\{h_{1}^{*}, h_{2}^{*}\right\}, d=$ $\frac{h_{3}^{*}}{\max \left\{h_{1}^{*}, h_{2}^{*}\right\}}\left(\delta^{*}-c\right) \widetilde{X}_{i}$ is the weighted average associated with population $\pi_{i}$.

The previous procedure would be meaningful only if the $a_{i j}$ exist. One can show the existence of the $a_{i j}$ 's through simple, but extended lines of algebra. Essentially what is being done on $a_{i j}$ 's here is an adjustment to allow for the fact that sample size must be a whole number, and that therefore a standard error estimate based on the preliminary sample takes only discrete values if all observations are equally weighted. By allocating unequal weights, the estimated standard error can be equated to a specific quantity.

Result: There exist $a_{i j}$ 's which satisfy:

$$
\begin{gathered}
\sum_{j=1}^{n_{i}} a_{i j}=1, \\
a_{i 1}=a_{i 2}=\cdots=a_{i n_{0}}, \\
S_{i}^{2} \sum_{j=1}^{n_{i}} a_{i j}^{2}=\left(\frac{\delta^{*}-c}{h^{*}}\right)^{2},
\end{gathered}
$$

where $i=1,2, \ldots, k$ and $j=1,2, \ldots, n_{i}$.

\section{Lower Bounds for $P\left(C S_{1}\right)$ and $P\left(C S_{2}\right)$}

To derive lower bounds for the probability of a correct selection, one needs to find the least favorable configuration as well as the worst configuration. We first define the least favorable configuration in the $P Z$ and the worst configuration in the $I Z$.

Definition 1 For any $\sigma^{2}=\left(\sigma_{1}^{2}, \sigma_{2}^{2}, \ldots, \sigma_{k}^{2}\right)$, the least favorable configuration in $P Z$ is defined to be: 


$$
L F C \mid P_{E}=\left\{\left(\mu_{0}, \sigma^{2}\right) \mid P\left(C S_{1} \mid\left(\mu_{0}, \sigma^{2}\right), P_{E}\right)=\inf _{\mu \in P Z} P\left(C S_{1} \mid\left(\mu, \sigma^{2}\right), P_{E}\right)\right\}
$$

Definition 2 For any $\sigma^{2}=\left(\sigma_{1}^{2}, \sigma_{2}^{2}, \ldots, \sigma_{k}^{2}\right)$, the worst configuration in IZ is defined to be:

$$
W C \mid P_{E}=\left\{\left(\mu_{1}, \sigma^{2}\right) \mid P\left(C S_{2} \mid\left(\mu_{1}, \sigma^{2} P_{E}\right)=\inf _{\mu \in I Z} P\left(C S_{2} \mid\left(\mu, \sigma^{2} P_{E}\right)\right)\right\} .\right.
$$

To derive lower bounds for $P\left(C S_{1}\right)$ and $P\left(C S_{2}\right)$ on the parameter space $\Omega$ we first show that (for any $\sigma^{2}$ ),

$$
L F C \mid P_{E}=\left\{\left(\mu, \sigma^{2}\right) \mid \delta_{k i}=\delta^{*} \quad \forall i \neq k\right\},
$$

where $\delta_{k i}=\mu_{[k]}-\mu_{[i]}$ and

$$
W C \mid P_{E}=\left\{\left(\mu, \sigma^{2}\right) \mid \delta_{k i}=0 \quad \forall i \neq k\right\} .
$$

Lemma 1 Let $T_{i}=\frac{\widetilde{X}_{(i)}-\mu_{[i]}}{\frac{\delta^{*}-c}{h^{*}}}$, then $T_{i}$ 's have independent student's $t$ distribution with $n_{0}-1$ degrees of freedom, $i=1,2, \ldots, k$.

Proof: The proof can be found in Stein (1945).

As the denominator $\left(s^{*}-c\right) / h^{*}$ is a constant, this lemma can only be true because the additional sample sizes $n_{i}$ are random.

Theorem 1 Under procedure $P_{E}$ the $L F C$ for $P\left(C S_{1} \mid P Z\right)$ is given by the slippage configuration, i.e. by $\mu_{[1]}=\cdots=\mu_{[k-1]}=\mu_{k}-\delta^{*}$ and the $W C$ for $P\left(C S_{2} \mid I Z\right)$ is given by the equal parameter configuration, i.e. by $\mu_{[1]}=\cdots=\mu_{[k]}$.

Proof: From (18), we find that the random variable $T_{i}(i=1,2, \ldots, k)$ has a $t$ - distribution with $n_{0}-1$ degrees of freedom.

Rewrite $\widetilde{X}_{I}$ as

$$
\tilde{X}_{(i)}=\left(\frac{\delta^{*}-c}{n^{*}}\right) T_{i}+\mu_{[i]}
$$

and consider the family of distribution function $\left\{\left(G_{n}(X \mid \mu)\right)\right\}$ where $G_{n}$ is the distribution of the random variable $\left(\frac{\delta^{*}-c}{n^{*}}\right) \cdot t_{n-1}+\mu$ where $\frac{\delta^{*}-c}{n^{*}}$ is a constant, $\mu$ is the parameter of interest, and $t_{n-1}$ is the random variable which has $t$ distribution with $n-1$ degrees of freedom. Then it is clear 
that $\left\{\left(G_{n}(X \mid \mu)\right)\right\}$ is a stochastically increasing family in $\mu$. We now show that the $L F C$ for $P\left(C S_{1} \mid P Z\right)$ is given by $\mu_{[1]}=\cdots=\mu_{[k-1]}=\mu_{[k]}-S^{*}$. The proof of the $W C$ for $P\left(C S_{2} \mid I Z\right)$ is similar. We start with an arbitrary configuration in the $I Z$

$$
\mu_{[1]} \leq \mu_{[1]} \leq \cdots \leq \mu_{[k]} \text { with } \mu_{[k]}-\mu_{\left[k_{1}\right]} S^{*}
$$

Letting $\bar{X}(i)$ denote the sample mean associated with $\mu_{[i]}$, we have

$$
P\left(C S_{1} \mid P Z\right)=P\left(\widetilde{X}_{(k)}>\max _{1 \leq \beta \leq k-1} \tilde{X}_{(\beta)}+C\right) .
$$

Define the function $\psi=\psi\left(y_{1}, y_{2}, \ldots, y_{k}\right)$ by

$$
\psi= \begin{cases}1 & \text { if } Y_{k}>\max _{l \leq \beta \leq k-t} y_{\beta}+C \\ 0 & \text { otherwise }\end{cases}
$$

Then we have $P\left(C S_{1} \mid P Z\right)=E \psi\left(\widetilde{X}_{(1)}, \widetilde{X}_{(2)}, \ldots, \widetilde{X}_{(k)}\right)$. It is clear that $\psi\left(y_{1}, y_{2}, \ldots, y_{k}\right)$ is non-increasing in $Y_{i}($ for $i=1, \ldots, k-1)$ when all the $y_{i}$ for $j \neq i$ are held fixed. Since $\widetilde{X}$ 's are from a stochastically increasing family, we use Lemma 5.1 by Chen and Sobel (1987) to conclude that $P\left(C S_{1} \mid P Z\right)$ is non-increasing in $\mu_{[i]}$ for $i=1,2, \ldots, k-1$ and it is nondecreasing in $u_{[k]}$. This completes the proof of the Theorem.

Lemma 2 Under procedure $P_{E}$, the probability of a correct selection in the $P Z$ and the $I Z$ are, respectively:

$$
\begin{aligned}
& P\left(C S_{1} \mid P_{E}\right)=P\left(\tilde{X}_{(k)} \geq \tilde{X}_{(i)}+c ; \quad i=1,2, \ldots, k-1\right), \\
& P\left(C S_{2} \mid P_{E}\right)=H_{0}+H_{1}+H_{2}
\end{aligned}
$$

where

$$
\begin{aligned}
H_{0}= & P\left(\widetilde{X}_{(k)} \geq M_{0}\right)=P\left(\widetilde{X}_{(k)} \geq \widetilde{X}_{(i)} ; \quad i=1,2, \ldots, k-1\right) \\
H_{1}= & P\left(M_{i} \leq \widetilde{X}_{(k)}<\widetilde{X}_{(i)}<\widetilde{X}_{(k)}+c, \quad i=1,2, \ldots, k-1\right) \\
= & \sum_{i=1}^{k-1} P\left(\widetilde{X}_{(i)}>\widetilde{X}_{(k)}>\widetilde{X}_{(j)}, \widetilde{X}_{(k)}+c>\widetilde{X}_{(i)}, j=1,2, \ldots, k-1, j \neq i\right) \\
H_{2}= & P\left(M_{i}-d \leq \widetilde{X}_{(k)} \leq M_{i} \leq \widetilde{X}_{(i)} \leq M_{i}+c, \quad i=1,2, \ldots, k-1\right) \\
& =\sum_{i=1}^{k-1} \sum_{j=1, j \neq i}^{k-1} P\left(\widetilde{X}_{(i)}>\widetilde{X}_{(j)}>\widetilde{X}_{(m)}, m=1,2, \ldots, k-1, m \neq i, j\right. \\
& \left.\widetilde{X}_{(j)}>\widetilde{X}_{(k)}>\widetilde{X}_{(j)}-d ; \widetilde{X}_{(j)}+c>\widetilde{X}_{(i)}\right) .
\end{aligned}
$$


and

$$
\begin{aligned}
M_{0} & =\max \left\{\tilde{X}_{(\alpha)} \mid \alpha=1,2, \ldots, k-1\right\}, \\
M_{i} & =\max \left\{\tilde{X}_{(\alpha)} \mid \alpha=1,2, \ldots, k-1, \alpha \neq i\right\} .
\end{aligned}
$$

Proof: The result is clear for $P\left(C S_{1} \mid P_{E}\right)$. For $P\left(C S_{2} \mid P_{s}\right), H_{0}, H_{1}$ and $H_{2}$ correspond to the cases of $\widetilde{X}_{(k)}$ being the largest, the second longest, and neither the largest nor the second largest, respectively.

The following theorems give lower bounds for $P\left(C S_{1} \mid P_{E}\right)$ and $P\left(C S_{2} \mid P_{E}\right)$.

Theorem 2 When $k=2$, for given $n_{0}$ and specification $\left(\delta^{*}, P_{1}^{*}, P_{2}^{*}, a\right)$, the $h_{1}^{*}$, and $h_{2}^{*}$ values which simultaneously satisfy:

$$
\begin{aligned}
& \int_{-\infty}^{+\infty} G\left(t+h_{1}^{*}\right) g(t) d t=P_{1}^{*}, \quad \text { and } \\
& \int_{-\infty}^{+\infty} G\left(t+\frac{h_{2}^{*}}{a-1}\right) g(t) d t=P_{2}^{*}
\end{aligned}
$$

are the values for procedure $P_{E}$ to satisfy the probability requirement (5). Here $G$ and $g$ are Student's t-distribution and density function, respectively.

Remark: When $k=2, d>0$ can be arbitrarily chosen since if we did not select the one best population, we would select two populations regardless the value of $d$.

Proof: Denote $\frac{\delta^{*}-c}{h^{*}}$ by $e^{*}$. By Lemma 2,

$$
\begin{aligned}
P\left(C S_{1} \mid P_{E}\right) & =P\left(\tilde{X}_{(2)} \geq \tilde{X}_{(1)}+c\right) \\
& =P\left(T_{1} \leq T_{2}+\frac{\delta_{21}-c}{e^{*}}\right) \\
& \geq P\left(T_{1} \leq T_{2}+h_{1}^{*}\right) \\
& =\int_{-\infty}^{\infty} G\left(t+h_{1}^{*}\right) g(t) d t=P_{1}^{*} .
\end{aligned}
$$

By Lemma 2, $P\left(C S_{2} \mid P_{E}\right)=H_{0}+H_{1}+H_{2}$. When $k=2$, the term $H_{2}$ does not exist. Thus

$$
\begin{aligned}
H_{0} & =P\left(\widetilde{X}_{(2)}>\widetilde{X}_{(1)}\right)=P\left(T_{1} \leq T_{2}+\frac{\delta_{21}}{e^{*}}\right) \\
H_{1} & =P\left(\widetilde{X}_{(2)}<\widetilde{X}_{(1)}, \widetilde{X}_{(1)}<\widetilde{X}_{(2)}+c\right) \\
& =P\left(\widetilde{X}_{(2)}<\widetilde{X}_{(1)}<\widetilde{X}_{(2)}+c\right) \\
& =P\left(T_{2}+\frac{\delta_{21}}{e^{*}} \leq T_{1}<T_{2}+\frac{\delta_{21}+c}{e^{*}}\right) .
\end{aligned}
$$


Therefore,

$$
\begin{aligned}
P\left(C S_{2} \mid P_{E}\right) & =H_{0}+H_{1} \\
& =P\left(T_{1}<T_{2}+\frac{\delta_{21}}{e^{*}}\right)+P\left(T_{2}+\frac{\delta_{21}}{e^{*}}<T_{1}<T_{2}+\frac{\delta_{21}+c}{e^{*}}\right) \\
& =P\left(T_{1}<T_{2}+\frac{\delta_{21}+c}{e^{*}}\right) \\
& \geq \int_{-\infty}^{\infty} G\left(t+\frac{c}{e^{*}}\right) g(t) d t \\
& \geq \int_{-\infty}^{\infty} G\left(t+\frac{h_{2}^{*}}{a-1}\right) g(t) d t=P_{2}^{*} .
\end{aligned}
$$

The first inequality follows from the fact that $T_{1}$ and $T_{2}$ both have students' $t$ distributions and $\delta_{21}=\mu_{[2]}-\mu_{[1]}$

From Theorem 2, it is clear that as $h_{1}^{*}, h_{2}^{*} \rightarrow \infty$, the left hand sides of (26) and (27) approach 1.

Theorem 3 For any $k \geq 3$ and any $n_{0}$ and specification $\left(\delta^{*}, P_{1}^{*}, P_{2}^{*}, a\right)$, the $h_{1}^{*}, h_{2}^{*}$ and $h_{3}^{*}$ values which simultaneously satisfy:

$$
\begin{gathered}
\int_{-\infty}^{+\infty} G^{k-1}\left(t+h_{1}^{*}\right) g(t) d t=P_{1}^{*}, \text { and } \\
\frac{1}{k}+(k-1) \int_{-\infty}^{+\infty} G^{k-2}(t)\left[G\left(t+\frac{h_{2}^{*}}{(a-1)}\right)-G(t)\right] g(t) d t \\
+(k-1)(k-2) \int_{-\infty}^{+\infty} G^{k-3}(t)\left[G\left(t+\frac{h_{2}^{*}}{(a-1)}\right)-G(t)\right] \\
\times\left[G(t)-G\left(t-h_{3}^{*}\right)\right] g(t) d t=P_{2}^{*}
\end{gathered}
$$

are the values for procedure $P_{E}$ to satisfy the probability requirement (5). Here $G$ and $g$ are Student's t-distribution and density function, respectively.

Proof: The proof of Theorem 3 is lengthy. It is omitted here. The readers may contact the first author for a full version of the manuscript which contains the proof.

The left hand side of the integral equations in (32) and in (33) in Theorem 3 are increasing in $h_{1}^{*}, h_{2}^{*}$ and $h_{3}^{*}$. Indeed, when $h_{1}^{*}$ approaches infinity, the left hand side of (32) increases to 1 . When $h_{2}^{*}$ and $h_{3}^{*}$ approach infinity, the left hand side of (33) also increases to 1 . Thus we can always find $h_{1}^{*}, h_{2}^{*}$, and $h_{3}^{*}$ that satisfy the probability requirements $P_{1}^{*}$ and $P_{2}^{*}$. 
One should note that it is necessary to let $h_{1}^{*}, h_{2}^{*}$ and $h_{3}^{*}$ vary freely so that our procedure will be applicable for any given probability requirements. Otherwise, the integral equations in (32) and in (33) might not have a solution, and in such a case, procedure $P_{E}$ is not applicable. For instance, if one requires $h_{1}^{*}=h_{2}^{*}$, then for some $\left(P_{1}^{*}, P_{2}^{*}\right)$ the integral equations in (32) and in (33) might not have a solution.

In procedure $P_{E}$, we let $\delta^{*}=a c, a>1$. Such a requirement has the advantage that the lower bounds of the probability of a correct selection do not involve $c$. Instead of letting $\delta^{*}=a c, a>1$, one can require that $\delta^{*}=a+c, a>0$. In such a case, (32) in Theorem 3 is unchanged. But (33) is changed to:

$$
\begin{aligned}
\frac{1}{k} & +(k-1) \int_{-\infty}^{+\infty} G^{k-2}(t)\left[G\left(t+\frac{h_{2}^{*} c}{a}\right)-G(t)\right] g(t) d t \\
& +(k-1)(k-2) \int_{-\infty}^{+\infty} G^{k-3}(t)\left[G\left(t+\frac{h_{2}^{*} c}{a}\right)-G(t)\right] \\
& \times\left[G(t)-G\left(t-h_{3}^{*}\right)\right] g(t) d t=P_{2}^{*} .
\end{aligned}
$$

\section{The Expected Sample Sizes and The Expected Subset Size}

The total sample size $n_{i}$ from population $\pi_{i}(i=1,2, \ldots, k)$ in procedure $P_{E}$ can be calculated from (7),

$$
n_{i}=\max \left\{n_{0}+1,\left[\left(\frac{S_{i} h^{*}}{\delta^{*}-c}\right)^{2}\right]\right\} .
$$

It is clear that $n_{i}, i=1,2, \ldots, k$, are random variables. The expected values of the sample sizes are often valuable to the experimenter. In our case, studying the expected sample size is especially important since there are two unknowns in the integral equation (11) with only one constraint. Thus we have infinitely many solutions. It is clear that we need some additional guidelines to choose $h_{2}^{*}$ and $h_{3}^{*}$. The expected sample size, which is a function of $h^{*}$, will give us some idea about how $h^{*}$ relates to $E\left(n_{i}\right)$. It is reasonable to choose $h_{2}^{*}$ and $h_{3}^{*}$ to minimize the expected sample sizes in addition to satisfying the probability requirements. To evaluate the expected sample sizes, we use the method of Stein (1945). 
Theorem 4 For any $i \in\{1,2, \ldots, k\}$, the expected sample size $E\left(n_{i}\right)$ for procedure $P_{E}$ satisfies the following inequality:

$$
\begin{aligned}
\left(n_{0}+1\right) & F_{n_{0}-1}\left(\frac{\left(n_{0}^{2}-1\right) e^{*^{2}}}{\sigma_{i}^{2}}\right)+\frac{\sigma_{i}^{2}}{e^{*^{2}}}\left[1-F_{n_{0}+1}\left(\frac{\left(n_{0}^{2}-1\right) e^{*^{2}}}{\sigma_{i}^{2}}\right)\right] \leq E\left(n_{i}\right) \\
& <\left(n_{0}+1\right) F_{n_{0}-1}\left(\frac{\left(n_{0}^{2}-1\right) e^{*^{2}}}{\sigma_{i}^{2}}\right)+\frac{\sigma_{i}^{2}}{e^{*^{2}}}\left[1-F_{n_{0}+1}\left(\frac{\left(n_{0}^{2}-1\right) e^{*^{2}}}{\sigma_{i}^{2}}\right)\right] \\
& +\left[1-F_{n_{0}-1}\left(\frac{\left(n_{0}^{2}-1\right) e^{*^{2}}}{\sigma_{i}^{2}}\right)\right]
\end{aligned}
$$

where $F_{i}(x)$ is a chi-squared probability distribution function with $i$ degrees of freedom and $e^{*^{2}}=\left(\frac{\delta^{*}-c}{h^{*}}\right)^{2}$.

Proof: The proofs follow the ideas of Stein (1945). It is omitted here. Readers are recommended to contact the first author for a full version of the transcript which contains the proof.

Corollary 1 For each $i, i=1,2, \ldots, k$, the expected sample size $E\left(n_{i}\right)$ has the following properties:

1. For fixed $e^{*^{2}}, E\left(n_{i}\right) \longrightarrow \infty$ as $\sigma_{i}^{2} \longrightarrow \infty$ (the lower bound of $E\left(n_{i}\right)$ goes to $+\infty)$.

2. For fixed $e^{*^{2}}, E\left(n_{i}\right) \longrightarrow n_{0}+1$ as $\sigma_{i}^{2} \longrightarrow 0$ (the upper bound of $E\left(n_{i}\right)$ goes to $\left.n_{0}+1\right)$.

3. For fixed $\sigma_{i}^{2}, E\left(n_{i}\right) \longrightarrow \infty$ as $e^{*^{2}} \longrightarrow 0$ (the lower bound of $E\left(n_{i}\right)$ goes to $+\infty)$.

4. The difference between the upper bounds and the lower bounds of $E\left(n_{i}\right)$ is at most 1 since $\left[1-F_{n_{0}-1}\left(\frac{\left(n_{0}^{2}-1\right) e^{*^{2}}}{\sigma_{i}^{2}}\right)\right]$ is less than 1 .

Proof: These properties are immediate by Theorem 4 .

\section{Tables}

To carry out procedure $P_{E}$, one needs the values of $h_{1}^{*}, h_{2}^{*}$, and $h_{3}^{*}$. In Table 1 , we provide a table of the $h_{1}^{\prime}$ value, for the cases $k=3,4$, which 
satisfies the following integral equation:

$$
\int_{-\infty}^{+\infty} G\left(t+h_{1}^{\prime}\right) g(t) d t=P^{*}
$$

for $P^{*}=.5, .75, .90, .95, .99$.

As discussed in section 4, there are infinitely many solutions for the integral equation (33). Therefore, it is impossible to provide tables which would cover all the practical situations. A particular solution of the integral equation (33) might be good for one objective yet might not be suitable for another goal.

Table 1. This table provides some $h_{1}^{\prime}$ values for procedure $P_{E}$.

\begin{tabular}{|r|r|r|r|r|r|}
\hline \multicolumn{7}{|c|}{ Number of populations: $k=3$} \\
\hline \multirow{2}{*}{$n_{0}$} & \multicolumn{5}{|c|}{ Probability $(\mathrm{P})$} \\
\cline { 2 - 6 } & .50 & .75 & .90 & .95 & .99 \\
\hline 3 & .7620 & 2.1560 & 4.0560 & 5.8750 & 13.1800 \\
\hline 4 & .6820 & 1.8650 & 3.2110 & 4.2840 & 7.4000 \\
\hline 5 & .6515 & 1.7390 & 2.8960 & 3.7500 & 5.9330 \\
\hline 6 & .6312 & 1.6700 & 2.7360 & 3.4810 & 5.2400 \\
\hline 7 & .6180 & 1.6260 & 2.6340 & 3.3180 & 4.8500 \\
\hline 8 & .6090 & 1.5960 & 2.5680 & 3.2100 & 4.6330 \\
\hline 9 & .6022 & 1.5740 & 2.5200 & 3.1340 & 4.4600 \\
\hline 10 & .5970 & 1.5578 & 2.4850 & 3.0746 & 4.3500 \\
\hline 11 & .5930 & 1.5445 & 2.4550 & 3.0370 & 4.2580 \\
\hline 12 & .5890 & 1.5318 & 2.4320 & 3.0060 & 4.1781 \\
\hline 13 & .5860 & 1.5240 & 2.4160 & 2.9800 & 4.1480 \\
\hline 14 & .5840 & 1.5180 & 2.4010 & 2.9560 & 4.0800 \\
\hline 15 & .5820 & 1.5128 & 2.3850 & 2.9360 & 4.0400 \\
\hline 20 & .5760 & 1.4900 & 2.3440 & 2.8560 & 3.8600 \\
\hline 25 & .5720 & 1.4770 & 2.3180 & 2.8260 & 3.8000 \\
\hline 30 & .5690 & 1.4700 & 2.3000 & 2.8000 & 3.7600 \\
\hline
\end{tabular}


Table 1. Continuation.

\begin{tabular}{|r|r|r|r|r|r|}
\hline \multicolumn{7}{|c|}{ Number of populations: $k=4$} \\
\hline$n_{0}$ & \multicolumn{5}{|c|}{ Probability $(\mathrm{P})$} \\
\cline { 2 - 6 } & .50 & .75 & .90 & .95 & .99 \\
\hline 3 & 1.1860 & 2.6615 & 4.7800 & 6.8200 & 15.1000 \\
\hline 4 & 1.0540 & 2.2500 & 3.6328 & 4.8000 & 8.2500 \\
\hline 5 & .9940 & 2.0810 & 3.2630 & 4.1360 & 6.0960 \\
\hline 6 & .9570 & 1.9880 & 3.0600 & 3.8150 & 5.6400 \\
\hline 7 & .9390 & 1.9310 & 2.9360 & 3.6160 & 5.2000 \\
\hline 8 & .9240 & 1.8920 & 2.8560 & 3.4980 & 4.9300 \\
\hline 9 & .9130 & 1.8630 & 2.7940 & 3.4100 & 4.7310 \\
\hline 10 & .9040 & 1.8410 & 2.7520 & 3.3450 & 4.6170 \\
\hline 11 & .8960 & 1.8230 & 2.7200 & 3.2960 & 4.5190 \\
\hline 12 & .8910 & 1.8100 & 2.6920 & 3.2580 & 4.4400 \\
\hline 13 & .8860 & 1.7970 & 2.6690 & 3.2280 & 4.3600 \\
\hline 14 & .8820 & 1.7890 & 2.6500 & 3.1980 & 4.3310 \\
\hline 15 & .8790 & 1.7820 & 2.6350 & 3.1760 & 4.2760 \\
\hline 20 & .8680 & 1.7530 & 2.5820 & 3.0880 & 4.0700 \\
\hline 25 & .8610 & 1.7380 & 2.5560 & 3.0450 & 4.0100 \\
\hline 30 & .8570 & 1.7280 & 2.5360 & 3.0220 & 3.960 \\
\hline
\end{tabular}

We tabulate in Table 2 the values of $h_{2}^{\prime}$ and $h_{3}^{\prime}$ for $k=3,4, P_{2}^{*}=$ $.50, .75, .90, .99$, where $h_{2}^{\prime}$ and $h_{3}^{\prime}$ satisfy the following integral equation:

$$
\begin{aligned}
& \frac{1}{k}+(k-1) \int_{-\infty}^{+\infty} G^{k-2}(t)\left[G\left(t+h_{2}^{\prime}\right)-G(t)\right] g(t) d t \\
& \quad+(k-1)(k-2) \int_{-\infty}^{+\infty} G^{k-3}(t)\left[G\left(t+h_{2}^{\prime}\right)-G(t)\right] \\
& \quad \times\left[G(t)-G\left(t-h_{3}^{\prime}\right)\right] g(t) d t=P_{2}^{*} .
\end{aligned}
$$

The relationship between $h_{2}^{*}, h_{3}^{*}$ and $h_{2}^{\prime}, h_{3}^{\prime}$ are as follows:

$$
h_{2}^{*}=(a-1) h_{2}^{\prime}, \quad h_{3}^{*}=h_{3}^{\prime} .
$$

The computation of Table 2 follows the following assumptions:

1. We take $a=2$ (thus, $\left.c=\frac{1}{2} \delta^{*}\right)$.

2. We take $h_{1}^{*}=h_{2}^{*}=h_{1}^{\prime}=h_{2}^{\prime}$ where $h_{1}^{\prime}$ is the value corresponding to $P_{1}^{*}=P_{2}^{*}=.50, .75, .90, .95, .99$ in Table 1 , respectively.

3. The probability is accurate to \pm .0003 . 
Table 2. This table provides some $\left(h_{2}, h_{3}\right)$ values for procedure $P_{E}$.

\begin{tabular}{|c|c|c|c|c|c|}
\hline \multicolumn{6}{|c|}{ Number of populations: $k=3$} \\
\hline \multirow[t]{2}{*}{$n_{0}$} & \multicolumn{5}{|c|}{ Probability $(\mathrm{P})$} \\
\hline & .50 & .75 & .90 & .95 & .99 \\
\hline \multirow[t]{2}{*}{3} & .7620 & 2.1560 & 4.0560 & 5.8750 & 13.1800 \\
\hline & .3860 & 1.2180 & 2.4850 & 3.8500 & 9.9600 \\
\hline \multirow[t]{2}{*}{4} & .6820 & 1.8650 & 3.2110 & 4.2840 & 7.4000 \\
\hline & .3550 & 1.0200 & 1.9450 & 2.7160 & 5.3000 \\
\hline \multirow[t]{2}{*}{5} & .6515 & 1.7390 & 2.8960 & 3.7500 & 5.9330 \\
\hline & .3260 & .9580 & 1.7480 & 2.3560 & 3.9100 \\
\hline \multirow[t]{2}{*}{6} & .6312 & 1.6700 & 2.7360 & 3.4810 & 5.2400 \\
\hline & .3210 & .9180 & 1.6380 & 2.1800 & 3.5000 \\
\hline \multirow[t]{2}{*}{7} & .6180 & 1.6260 & 2.6340 & 3.3180 & 4.8500 \\
\hline & .3160 & .8940 & 1.5830 & 2.1000 & 3.3600 \\
\hline \multirow[t]{2}{*}{8} & .6090 & 1.5960 & 2.5680 & 3.2100 & 4.6330 \\
\hline & .3100 & .8745 & 1.5320 & 2.0160 & 3.1300 \\
\hline \multirow[t]{2}{*}{9} & .6022 & 1.5740 & 2.5200 & 3.1340 & 4.4600 \\
\hline & .3060 & .8570 & 1.5030 & 1.9650 & 3.0300 \\
\hline \multirow[t]{2}{*}{10} & .5970 & 1.5578 & 2.4850 & 3.0746 & 4.3500 \\
\hline & .3050 & .8500 & 1.4800 & 1.9460 & 2.9200 \\
\hline \multirow[t]{2}{*}{11} & .5930 & 1.5445 & 2.4550 & 3.0370 & 4.2580 \\
\hline & .3020 & .8420 & 1.4620 & 1.9160 & 2.8800 \\
\hline \multirow[t]{2}{*}{12} & .5890 & 1.5318 & 2.4320 & 3.0060 & 4.1781 \\
\hline & .2990 & .8360 & 1.4500 & 1.8830 & 2.7700 \\
\hline \multirow[t]{2}{*}{13} & .5860 & 1.5240 & 2.4160 & 2.9800 & 4.1480 \\
\hline & .2970 & .8300 & 1.4360 & 1.8680 & 2.8000 \\
\hline \multirow[t]{2}{*}{14} & .5840 & 1.5180 & 2.4010 & 2.9560 & 4.0800 \\
\hline & .2950 & .8260 & 1.4250 & 1.8400 & 2.7600 \\
\hline \multirow[t]{2}{*}{15} & .5820 & 1.5128 & 2.3850 & 2.9360 & 4.0400 \\
\hline & .2930 & .8210 & 1.4180 & 1.8390 & 2.7400 \\
\hline \multirow[t]{2}{*}{20} & .5760 & 1.4900 & 2.3440 & 2.8560 & 3.8600 \\
\hline & .2900 & .8080 & 1.3900 & 1.8060 & 2.6800 \\
\hline \multirow[t]{2}{*}{25} & .5720 & 1.4770 & 2.3180 & 2.8260 & 3.8000 \\
\hline & .2880 & .8030 & 1.3810 & 1.7900 & 2.6150 \\
\hline \multirow[t]{2}{*}{30} & .5690 & 1.4700 & 2.3000 & 2.8000 & 3.7600 \\
\hline & .2879 & .8000 & 1.3660 & 1.7730 & 2.6140 \\
\hline
\end{tabular}

Note: Here we let $h_{2}^{\prime}=h_{1}^{\prime}$. 
Table 2. Continuation.

\begin{tabular}{|c|c|c|c|c|c|}
\hline \multicolumn{6}{|c|}{ Number of populations: $k=4$} \\
\hline \multirow{2}{*}{$n_{0}$} & \multicolumn{5}{|c|}{ Probability $(\mathrm{P})$} \\
\hline & .50 & .75 & .90 & .95 & .99 \\
\hline \multirow[t]{2}{*}{3} & 1.1860 & 2.6615 & 4.7800 & 6.8200 & 15.1000 \\
\hline & .6500 & 1.6220 & 3.1200 & 4.5800 & 12.0000 \\
\hline \multirow[t]{2}{*}{4} & 1.0540 & 2.2500 & 3.6328 & 4.8000 & 8.2500 \\
\hline & .5760 & 1.3620 & 2.3750 & 3.2000 & 12.9000 \\
\hline \multirow[t]{2}{*}{5} & .9940 & 2.0810 & 3.2630 & 4.1360 & 6.0960 \\
\hline & .5410 & 1.2560 & 2.1030 & 2.7720 & 4.6500 \\
\hline \multirow[t]{2}{*}{6} & 570 & 1.9880 & 3.0600 & 3.8150 & 5.6400 \\
\hline & .5260 & 1.1990 & 1.9920 & 2.5170 & 3.9950 \\
\hline \multirow[t]{2}{*}{7} & .9390 & 1.9310 & 2.9360 & 3.6160 & 5.2000 \\
\hline & .5100 & 1.1 & 1.9200 & 2.4640 & 7960 \\
\hline \multirow[t]{2}{*}{8} & .9240 & 1.8920 & 2.8560 & 3.4980 & 4.9300 \\
\hline & .5000 & 1.13 & 1.8660 & 2.3780 & 300 \\
\hline \multirow[t]{2}{*}{9} & .9130 & 1.8630 & 2.7940 & 3.4100 & 4.7310 \\
\hline & .4935 & 1.1190 & 1.8260 & 2.3190 & 3.4000 \\
\hline \multirow[t]{2}{*}{10} & .9040 & 1.84 & 2.7520 & 3.3450 & 4.6170 \\
\hline & .4880 & 1.1050 & 1.8000 & 2.2800 & 3.2900 \\
\hline \multirow[t]{2}{*}{11} & .8960 & 1.8230 & 2.7200 & 3.2960 & 4.5190 \\
\hline & .4810 & 1.0 & 1.7800 & 2.2480 & 3.2500 \\
\hline \multirow[t]{2}{*}{12} & 910 & 1.81 & 2.6920 & 3.2580 & 4.4400 \\
\hline & .4780 & 1.08 & 1.7560 & 2.2200 & 3.2050 \\
\hline \multirow[t]{2}{*}{13} & .8860 & 1.7970 & 2.6690 & 3.2280 & 4.3600 \\
\hline & .4776 & 1.0780 & 1.7500 & 2.1900 & 3.1450 \\
\hline \multirow[t]{2}{*}{14} & .8820 & 1.7890 & 2.6500 & 3.1980 & 4.3310 \\
\hline & .4760 & 1.0370 & 1.7380 & 2.1860 & 3.1400 \\
\hline \multirow[t]{2}{*}{15} & .8790 & 1.7820 & 2.6350 & 3.1760 & 4.2760 \\
\hline & .4730 & & 1.7300 & 2.1750 & 3.1130 \\
\hline \multirow[t]{2}{*}{20} & .8680 & 1.7530 & 2.5820 & 3.0880 & 4.0700 \\
\hline & .4670 & 1.0520 & 1.6980 & 2.1300 & 3.0330 \\
\hline \multirow[t]{2}{*}{25} & .8610 & 7380 & 2.5560 & 3.0450 & 4.0100 \\
\hline & .4660 & 1.0430 & 1.6840 & 2.1030 & 3.0200 \\
\hline \multirow[t]{2}{*}{30} & .8570 & 1.7280 & 2.5360 & 3.0220 & 3.9600 \\
\hline & .4640 & 1.0380 & 1.6720 & 2.1000 & 2.9800 \\
\hline
\end{tabular}

We use Fortran77 to program the double integrals. Integration is carried out by the Romberg numerical method (Burden and Faires (1988)) in which 
Neville's algorithm (Burden and Faires (1988)) is used for extrapolation. We modified the subroutines provided by Press, Teukolsky, Vetterling, and Flannery (1992) for our program. The upper limits of the integration for the student's $t$-density functions depend on the degree of freedom of the density function. All real variables are declared as double precision. Programs are executed under a UNIX environment using SUN4 600 Series and SUN4 Sparc 2000 machines.

We also provide a table (Table 3 ) of the approximation for the expected sample sizes using the $h=h_{1}^{\prime}$ value obtained in Table 1 and for $r=$ $\frac{\left(\delta^{*}-c\right)^{2}}{\sigma_{i}^{2}}$. Mathematica was used to perform the calculation. We compute the approximation of the expected sample sizes using the lower bound formula for $E\left(n_{i}\right)$ in Theorem 4 . The formula is:

$$
\left(n_{0}+1\right) F_{n_{0}-1}\left(\frac{\left(n_{0}^{2}-1\right) r}{h^{2}}\right)+\frac{h^{2}}{r}\left[1-F_{n_{0}+1}\left(\frac{\left(n_{0}^{2}-1\right) r}{h^{2}}\right)\right] .
$$

By (38), it is clear that $E\left(n_{i}\right)$ is dominated by $\frac{h^{2}}{r}$ when $r$ is small, $h$ is large, and $n_{0}$ is not very large. Indeed, from Table 3 one sees that the change of $E\left(n_{i}\right)$ is proportional to the change of $r$ for a fixed $h$ and when $r$ is small, $h$ is large, and $n_{0}$ is not very large. In fact $\frac{h^{2}}{r}$ is a precise estimate of $E\left(n_{i}\right)$ when $r$ is small, $h$ is large, and $n_{0}$ is not very large.

Table 3. This table provides some approximations of the expected sample sizes for procedure $P_{E}$.

\begin{tabular}{|r|r|r|r|r|r|r|r|r|r|}
\hline \multicolumn{10}{|c|}{$k=3, P_{1}^{*}=.90$} \\
\hline$n_{0}$ & \multicolumn{10}{|c|}{$\mathrm{r}$} & \multicolumn{1}{|c|}{} \\
\cline { 2 - 10 } & .05 & .10 & .30 & .45 & .60 & .75 & 1.0 & 1.25 & 1.5 \\
\hline 3 & 329.047 & 164.560 & 54.980 & 36.769 & 27.697 & 22.278 & 16.900 & 13.712 & 11.616 \\
\hline 4 & 206.210 & 103.105 & 34.374 & 22.930 & 17.222 & 13.814 & 10.445 & 8.472 & 7.205 \\
\hline 6 & 149.714 & 74.857 & 24.961 & 16.681 & 12.606 & 10.252 & 8.040 & 7.046 & 6.514 \\
\hline 8 & 131.892 & 65.946 & 22.002 & 14.794 & 11.429 & 9.705 & 8.493 & 8.129 & 8.031 \\
\hline 10 & 123.505 & 61.760 & 20.993 & 14.961 & 12.610 & 11.648 & 11.136 & 11.027 & 11.005 \\
\hline 15 & 113.765 & 56.882 & 19.474 & 15.541 & 15.036 & 15.001 & 15.000 & 15.000 & 15.000 \\
\hline 20 & 109.887 & 54.944 & 21.006 & 20.010 & 20.000 & 20.000 & 20.000 & 20.000 & 20.000 \\
\hline 25 & 107.462 & 53.732 & 25.069 & 25.000 & 25.000 & 25.000 & 25.000 & 25.000 & 25.000 \\
\hline
\end{tabular}


Table 3. Continuation.

\begin{tabular}{|r|r|r|r|r|r|r|r|r|r|}
\hline \multicolumn{10}{|c|}{$k=3, P_{1}^{*}=.95$} \\
\hline \multirow{2}{*}{$n_{0}$} & \multicolumn{8}{|c|}{$\mathrm{r}$} \\
\cline { 2 - 11 } & .05 & .10 & .30 & .45 & .60 & .75 & 1.0 & 1.25 & 1.5 \\
\hline 3 & 690.324 & 345.179 & 115.121 & 76.804 & 57.662 & 46.190 & 34.739 & 27.889 & 23.339 \\
\hline 4 & 367.053 & 183.527 & 61.177 & 40.787 & 30.596 & 24.485 & 18.384 & 14.739 & 12.324 \\
\hline 6 & 242.347 & 121.174 & 40.392 & 26.934 & 20.217 & 16.208 & 12.261 & 9.990 & 8.582 \\
\hline 8 & 206.082 & 103.041 & 34.349 & 22.914 & 17.242 & 13.922 & 10.853 & 9.341 & 8.606 \\
\hline 10 & 189.113 & 94.558 & 31.621 & 21.397 & 16.632 & 14.119 & 12.169 & 11.430 & 11.155 \\
\hline 15 & 172.402 & 86.201 & 28.763 & 19.641 & 16.249 & 15.254 & 15.010 & 15.000 & 15.000 \\
\hline 20 & 163.135 & 81.567 & 27.456 & 20.932 & 20.050 & 20.001 & 20.000 & 20.000 & 20.000 \\
\hline 25 & 159.726 & 79.863 & 28.024 & 25.060 & 25.000 & 25.000 & 25.000 & 25.000 & 25.000 \\
\hline
\end{tabular}

\begin{tabular}{|r|r|r|r|r|r|r|r|r|r|}
\hline \multicolumn{10}{|c|}{$k=3, P_{1}^{*}=.99$} \\
\hline$n_{0}$ & \multicolumn{10}{|c|}{$\mathrm{r}$} & \multicolumn{1}{|c|}{} \\
\cline { 2 - 10 } & .05 & .10 & .30 & .45 & .60 & .75 & 1.0 & 1.25 & 1.5 \\
\hline 3 & 3474.250 & 1737.130 & 579.055 & 386.048 & 289.548 & 231.651 & 173.758 & 143.027 & 115.877 \\
\hline 4 & 1095.200 & 547.600 & 182.533 & 121.689 & 91.267 & 73.014 & 54.761 & 43.811 & 36.512 \\
\hline 6 & 549.152 & 274.576 & 91.525 & 61.017 & 45.763 & 36.612 & 27.464 & 21.981 & 18.337 \\
\hline 8 & 429.294 & 214.647 & 71.549 & 47.700 & 35.776 & 28.625 & 21.488 & 17.240 & 14.464 \\
\hline 10 & 378.450 & 189.225 & 63.083 & 42.086 & 31.639 & 25.446 & 19.448 & 16.107 & 14.124 \\
\hline 15 & 326.432 & 163.216 & 54.405 & 36.274 & 27.248 & 21.986 & 17.425 & 15.862 & 15.155 \\
\hline 20 & 297.992 & 148.996 & 49.666 & 33.160 & 25.349 & 21.748 & 20.152 & 20.007 & 20.000 \\
\hline 25 & 288.800 & 144.400 & 48.138 & 32.441 & 26.546 & 25.178 & 25.002 & 25.000 & 25.000 \\
\hline
\end{tabular}

\begin{tabular}{|r|r|r|r|r|r|r|r|r|r|}
\hline \multicolumn{10}{|c|}{$k=4, P_{1}^{*}=.90$} \\
\hline$n_{0}$ & \multicolumn{10}{|c|}{$\mathrm{r}$} & \multicolumn{10}{|c|}{} \\
\cline { 2 - 11 } & .05 & .10 & .30 & .45 & .60 & .75 & 1.0 & 1.25 & 1.5 \\
\hline 3 & 456.985 & 228.519 & 76.265 & 50.928 & 38.284 & 30.716 & 23.179 & 18.686 & 15.714 \\
\hline 4 & 263.974 & 131.987 & 43.999 & 29.339 & 22.017 & 17.633 & 13.273 & 10.686 & 8.993 \\
\hline 6 & 187.272 & 93.636 & 31.216 & 20.827 & 15.664 & 12.615 & 9.693 & 8.108 & 7.206 \\
\hline 8 & 163.135 & 81.567 & 27.196 & 18.179 & 13.786 & 11.330 & 9.287 & 8.467 & 8.158 \\
\hline 10 & 151.470 & 75.739 & 25.461 & 17.561 & 14.130 & 12.502 & 11.433 & 11.120 & 11.032 \\
\hline 15 & 138.865 & 69.432 & 23.292 & 16.844 & 15.270 & 15.027 & 15.000 & 15.000 & 15.000 \\
\hline 20 & 133.334 & 66.667 & 23.264 & 20.143 & 20.002 & 20.000 & 20.000 & 20.000 & 20.000 \\
\hline 25 & 130.663 & 65.331 & 25.670 & 25.001 & 25.000 & 25.000 & 25.000 & 25.000 & 25.000 \\
\hline
\end{tabular}


Table 3. Continuation.

\begin{tabular}{|r|r|r|r|r|r|r|r|r|r|}
\hline \multicolumn{10}{|c|}{$k=4, P_{1}^{*}=.95$} \\
\hline$n_{0}$ & \multicolumn{10}{|c|}{$\mathrm{r}$} \\
\cline { 2 - 11 } & .05 & .10 & .30 & .45 & .60 & .75 & 1.0 & 1.25 & 1.5 \\
\hline 3 & 930.257 & 465.141 & 155.092 & 103.437 & 77.622 & 62.143 & 46.680 & 37.417 & 31.256 \\
\hline 4 & 460.800 & 230.400 & 76.801 & 51.202 & 38.404 & 30.728 & 23.057 & 18.463 & 15.410 \\
\hline 6 & 291.085 & 145.542 & 48.515 & 32.346 & 24.267 & 19.431 & 14.630 & 11.808 & 9.998 \\
\hline 8 & 244.720 & 122.360 & 40.787 & 27.198 & 20.423 & 16.401 & 15.529 & 10.441 & 9.288 \\
\hline 10 & 223.781 & 111.891 & 37.353 & 25.091 & 19.196 & 15.924 & 13.250 & 11.935 & 11.401 \\
\hline 15 & 201.740 & 100.870 & 33.631 & 22.600 & 17.771 & 15.831 & 15.072 & 15.004 & 15.000 \\
\hline 20 & 190.715 & 95.357 & 31.858 & 22.549 & 20.299 & 20.020 & 20.000 & 20.000 & 20.000 \\
\hline 25 & 185.441 & 92.720 & 31.389 & 25.386 & 25.006 & 25.000 & 25.000 & 25.000 & 25.000 \\
\hline
\end{tabular}

\begin{tabular}{|r|r|r|r|r|r|r|r|r|r|}
\hline \multicolumn{10}{|c|}{$k=4, \quad P_{1}^{*}=.99$} \\
\hline$n_{0}$ & \multicolumn{10}{|c|}{$\mathrm{r}$} & \multicolumn{1}{|c|}{} \\
\cline { 2 - 10 } & .05 & .10 & .30 & .45 & .60 & .75 & 1.0 & 1.25 & 1.5 \\
\hline 3 & 4560.200 & 2280.100 & 760.044 & 605.705 & 380.038 & 304.040 & 228.045 & 182.452 & 152.059 \\
\hline 4 & 1361.250 & 680.625 & 226.875 & 151.250 & 113.438 & 90.750 & 68.063 & 54.452 & 45.378 \\
\hline 6 & 636.192 & 318.096 & 106.032 & 70.688 & 53.016 & 42.414 & 31.813 & 25.456 & 21.224 \\
\hline 8 & 486.098 & 243.049 & 81.016 & 54.011 & 40.509 & 32.409 & 24.317 & 19.482 & 16.293 \\
\hline 10 & 426.334 & 213.167 & 71.606 & 47.393 & 35.595 & 28.568 & 21.685 & 17.758 & 15.348 \\
\hline 15 & 365.684 & 182.842 & 60.947 & 40.633 & 30.492 & 24.482 & 18.911 & 16.384 & 15.412 \\
\hline 20 & 331.298 & 165.649 & 55.217 & 36.828 & 27.845 & 23.166 & 20.442 & 20.035 & 20.002 \\
\hline 25 & 321.602 & 160.801 & 53.601 & 35.865 & 28.145 & 25.577 & 25.012 & 25.000 & 25.000 \\
\hline
\end{tabular}

\section{An Illustrative Example}

Now we present an example to illustrate the procedure $P_{E}$.

Example: Suppose that we are given three normal populations with unequal and unknown variances. Suppose that we wish to use the integrated formulation to select the population having the largest population mean if $\mu_{[3]}-\mu_{[2]} \geq 1$, and to select a subset that contains the longest mean if $\mu_{[3]}-\mu_{[2]}<1$.

Suppose that for certain practical reasons, the experimenter decides to take a initial sample of size $n_{0}=15$. We use Fortran to generate three random samples of size 15 from population $N\left(4, .9^{2}\right), N\left(4.5,1^{2}\right)$, and $N\left(5.5,1.5^{2}\right)$. 
We obtain:

$$
\begin{aligned}
& \sum_{j=1}^{15} X_{1 j}=57.4729, \sum_{j=1}^{5} X_{2 j}=63.6917, \sum_{j=1}^{5} X_{3 j}=89.5628 \text {, } \\
& S_{1}(15)=.76247, \quad S_{2}(15)=.82931, \quad S_{3}(15)=1.2974 .
\end{aligned}
$$

Now we suppose that the experimenter has specified $P_{1}^{*}=P_{2}^{*}=.95$ and $\delta^{*}=1$. Suppose that the experimenter also specified $a=2$ (i.e. $c=\frac{1}{2}$ ). From Table 1 with $k=3, n_{0}=15$, and $P_{1}^{*}=.95$, the experimenter finds $h_{1}^{*}=2.9360$. From Table 2 with $k=3, n_{0}=15$ and $P_{2}^{*}=.95$, the experimenter finds that $h_{2}^{\prime}=2.9360, h_{3}^{\prime}=1.839$. Therefore, $h_{2}^{*}=$ $(a-1) h_{2}^{\prime}=2.9360$ and $h_{3}^{*}=h_{3}^{\prime}=1.839$. Thus the experimenter finds that $h^{*}=\max \left\{h_{1}^{*}, h_{2}^{*}\right\}=2.9360$ (here $h_{1}^{*}$ and $h_{2}^{*}$ are the same since we choose them to be the same (when $a=2$ ) in the calculation of Table 2), and

$$
n_{i}=\max \left\{16,\left[\left(\frac{S_{i} \times 2.9360}{1-\frac{1}{2}}\right)^{2}\right]\right\} \text {. }
$$

We obtain $n_{1}=21, n_{2}=24$, and $n_{3}=59$. Hence 6 , 9, and 44 additional observations must be taken from population one, two, and three, respectively. The experimenter also computes $d=1.839 \times \frac{\frac{1}{2}}{2.9360}=0.3132$. Therefore, the selection rule is:

select the population associated with $\bar{X}_{[3]}$ if $\bar{X}_{[3]} \geq \bar{X}_{[2]}+.5$, or

select the populations which satisfy $\bar{X}_{(i)} \geq \bar{X}_{[2]}-.3132$ if $\bar{X}_{[3]}<\bar{X}_{[2]}+.5$.

The Fortran program generates the second samples of appropriate size from populations $N\left(4, .9^{2}\right), N\left(4.5,1^{2}\right)$, and $N\left(5.5,1.5^{2}\right)$, respectively. In order to compute the weighted averages, one needs to specify the weights $a_{i j}, i=1,2,3 ; j=1,2, \ldots, n_{i}$ which would satisfy the conditions (13) and (14). To specify the $a_{i j}$ 's, we first compute:

$$
c_{i}=\frac{\left(n_{i}-1\right)+\sqrt{\left(n_{i}-1\right)\left[\left(n_{i}-1\right)-n_{i}\left(1-\frac{e^{2}}{S_{i}^{2}}\right)\right]}}{\left(n_{i}-1\right) n_{i}} .
$$

By letting $a_{i j}=c_{i}, i=1,2,3 ; j=1,2, \ldots, n_{i}-1$ and $a_{i n_{i}}=1-c_{i}\left(n_{i}-\right.$ $1), i=1,2,3$, we are guaranteed that the conditions (13) and (14) are satisfied. Our program computes $c_{1}=.0499423, c_{2}=.0426206$, and $c_{3}=.0172361$. Therefore, $a_{1 j}=.0499423, j=1,2, \ldots, 20, a_{1,21}=.00154$; $a_{2 j}=.0426206, j=1,2, \ldots, 23, a_{2,24}=.0197262 ; a_{3 j}=.0172361, j=$ 
$1,2, \ldots, 58, a_{3,59}=.0003062$. One can easily check that $S_{i}^{2} \sum_{j=1}^{n_{i}} a_{i j}=$ $\left(\frac{1}{2 h_{2}^{*}}\right)^{2}=.0290$, for $i=1,2,3$. The weighted averages are:

$$
\bar{X}_{1}=3.95310, \quad \bar{X}_{2}=4.37875, \quad \bar{X}_{3}=5.44820 .
$$

Since $\bar{X}_{[2]}+.5=4.37875+.5=4.87875$ and $\bar{X}_{[3]}=5.44820>4.87875$, the experimenter will select only the population number three and claim that its weight is the largest.

\section{References}

1. R. E. Bechhofer. A single-sample multiple decision procedure for ranking means of normal populations with known variances. Annals of Mathematical Statistics, 25:1639, 1954.

2. R. E. Bechhofer, C. W. Dunnett, and M. Sobel. A two sample multiple decision procedure for ranking means of normal populations with a common unknown variance. Biometrika, 41:170-176, 1954.

3. R. L. Burden, and J. D. Faires. Numerical Analysis, 4th Edition. PWS-Kent Publishing Co., 1988.

4. P. Chen and M. Sobel. An integrated formulation for selecting the $t$ best of $k$ normal populations. Communications in Statistics, Theory and Methods, 16(1):121146, 1987.

5. P. Chen and J. Zhang. An integrated formulation for selecting the best normal populations: the common and unknown variance case. Communications in Statistics, Theory and Methods, 26(11): 2701- 2724, 1997.

6. E. J. Dudewicz. Non-existence of a single-sample selection procedure whose $P(C S)$ is independent of the variances. South Africa Statistics Journal, 5:37-39, 1971.

7. E. J. Dudewicz and S. R. Dalal. Allocation of observations in ranking and selection with unequal variances. Sankhya, Series A:28-78, 1975.

8. S. S. Gupta and W. T. Huang. A note on selecting a subset of normal populations with unequal sample sizes. Sankhya, Series A:389-396, 1974.

9. W. H. Press, S. A. Teukolsky, W. T. Vetterling, and B. P. Flannery. Numerical recipes in FORTRAN. Cambridge University Press, 1992.

10. C. M. Stein. A two sample test for a linear hypothesis whose power is independent of the variance. Annals of Mathematical Statistics, 16:243-258, 1945. 